\title{
Erasing of an optically induced grating in a semi-insulating multiple quantum well waveguide
}

\author{
Ewa Weinert-Rączka and Marek Wichtowski \\ Faculty of Electrical Engineering, West Pomeranian University of Technology in Szczecin, Al. Piastów 17, \\ 70-310 Szczecin, Poland
}

Received March 12, 2012; accepted March 28, 2012; published March 31, 2012

\begin{abstract}
Interaction is considered between optical signal guided in a photorefractive multiple quantum well planar waveguide and two external waves. The external waves create a refractive index grating in the waveguide core which can be applied for frequency selective switching of guided signals. The influence is analyzed of free carriers generated by the signal wave on the stability of photorefractive grating.
\end{abstract}

Semi-insulating multiple quantum wells (MQW) have strong electro-optic properties and short response time compared with bulk photorefractive materials. Therefore they seem to be a promising material for applications in electro-optics devices, especially as optical switchers and modulators. In the last few years several applications of photorefractive MQW have been proposed and demonstrated [1]. The electro-optic properties of photorefractive MQW (PMQW) are related to excitonic transitions and therefore accompanied by strong absorption. Usually a few micrometers thick PMQW device operates in transmission geometry with light beams of resonant frequency. One of the typical transmission geometry experiments is photorefractive two-wave mixing (TWM) [2].

For optical signals of frequency detuned from the resonance absorption can be significantly reduced and PMQW structure can be used as a guiding layer of a planar waveguide [3]. Such geometry gives a lot of new possibilities including generation of screening solitons $[4,5]$ and different interactions between resonant external beams and off-resonant guided signals. For example, the photorefractive grating created by two external beams in a TWM experiment can influence the signal propagating in a PMQW waveguide leading to frequency selective reflection, deflection or outcoupling [6].

In the case of resonant frequency beams the dominant mechanism of free carriers generation relies on direct transitions and the influence of photoionization of deep impurities is not taken into account. The signal wave does not participate in direct transitions, but due to large intensity resulting from the confinement in the waveguide, causes photoionization of deep defects. Here we investigate, for the first time according to our knowledge, the influence of this process on the stability of the grating created by two resonant external beams.
The analyzed device is a single-mode planar waveguide containing a semi-insulating multiple quantum well guiding layer. The MQW structure operates in socalled Franz - Keldysh geometry with the external electric field applied along the quantum well planes [7-10]. Two mutually coherent external beams of intensities $I_{w 1}$ and $I_{w 2}$ overlap in the MQW layer and create the intensity pattern described by :

$$
I_{w}(z)=I_{0}[1+m \cos (K z)],
$$

where $I_{0}=I_{w 1}+I_{w 2}$ is total light intensity of the writing beams and $m$ denotes the modulation depth. The grating vector $K$ depends on the wavelengths and propagation directions of writing beams.

Processes leading to the photorefractive effect in semiinsulating MQW operating in the Franz - Keldysh geometry are similar as in bulk materials $[1,7]$. The high frequency light of external beams creates electron-hole pairs by direct interband transitions. The carriers are excited in bright fringes of the interference pattern, move due to the drift and diffusion and recombine to the deep traps mainly in the dark regions, creating a spatially modulated charge distribution. A non-uniform charge distribution builds up the space-charge electric field. This field changes the refractive index through an electro-optic effect. The resulting refractive index grating can influence signals propagating in the waveguide.

The photorefractive transport model including the hotelectron effect is based on the scheme proposed in the paper [11]. In this approach for electrons is attributed temperature $T_{e}(E)$ which can be significantly higher under a large electric field intensity $E$ than the temperature of the lattice $T_{L}$. As a consequence, the electron mobility $\mu_{e}(E)$ and the velocity of electrons $v_{e}(E)$ depend on the electric field intensity $E$ while the hole transport is assumed to remain linear even at a high electric field $[7,11]$. For the sake of simplicity we assumed that the impurity levels in the MQW structure are described by one deep-level of donor traps of concentration $N_{D}$ and one shallow-level of acceptors of concentration $N_{A}$ which compensate donors [7],[9-11]. The acceptors are completely ionized $\left(N_{A}=N_{A}^{-}\right)$and do not participate in 
the photorefractive process but ensure initial concentration of the ionized deep donors $N_{D}^{+}(t=0)=N_{A}^{-}$. If photogenerated carriers are confined in quantum wells, the model can be reduced to the set of one-dimensional equations describing processes of carrier generation, transport and recombination. Neglecting the direct dark generation, direct recombination and thermal excitation of electrons and holes the photorefractive transport equations are [11]:

$$
\begin{aligned}
& \frac{\partial n_{e}}{\partial t}=\left(b+b_{e w}\right) I_{w}+b_{e s} I_{s}-\gamma_{e} n_{e} N_{D}^{+}+\frac{1}{q} \frac{\partial j_{e}}{\partial z}, \\
& \frac{\partial n_{h}}{\partial t}=\left(b+b_{h w}\right) I_{w}+b_{h s} I_{s}-\gamma_{h} n_{h} N_{D}^{0}-\frac{1}{q} \frac{\partial j_{h}}{\partial z}, \\
& \frac{\partial \rho}{\partial t}=-\frac{\partial}{\partial z}\left(j_{e}+j_{h}\right), \\
& j_{e}=q \mu_{e}(E) E n_{e}+k_{B} \frac{\partial}{\partial z}\left(\mu_{e}(E) T_{e}(E) n_{e}\right), \\
& j_{h}=q \mu_{h} E n_{h}-k_{B} \mu_{h} T_{L} \frac{\partial n_{h}}{\partial z}, \\
& \frac{\partial E}{\partial z}=\frac{\rho}{\varepsilon_{0} \varepsilon}, \\
& \rho=q\left(N_{D}^{+}-N_{A}^{-}+n_{h}-n_{e}\right) .
\end{aligned}
$$

where the subscripts $e$ and $h$ mark electron and hole properties respectively, $n$ denotes the free carriers concentration, $N_{D}$ - donors, $N_{D}^{+}$- ionized donors and $N_{A}-$ acceptors concentration, $j-$ the current density, $E-$ the total electric field $\left(E=E_{a}+E_{s c}\right.$, where $E_{a}$ is an external generation of free carriers rate, terms $b_{i w} I_{w}$ and $b_{i s} I_{s}(i=$ $e, h)$ - photoionization of defects rate, where

$$
b=\frac{\alpha}{h v_{w}}, b_{e j}=\frac{\alpha_{e}}{h v_{j}}=\frac{s_{e}\left(N_{D}-N_{A}\right)}{h v_{j}}, \quad b_{h j}=\frac{\alpha_{h}}{h v_{j}}=\frac{s_{h} N_{A}}{h v_{j}},
$$

$j=w, s\left(w-\right.$ writing wave, $s-$ signal wave), $h v_{j}$ are photon energies, $\alpha$ denotes the absorption coefficients due to the generation of electron-hole pairs, $\alpha_{e}, \alpha_{h}-$ absorption due to the generation of free electrons and holes from defect levels.

The absorption coefficient $\alpha$ for the writing beams is of the order of $10^{4} \mathrm{~cm}^{-1}$ [7]-[9], while the absorption coefficients $\alpha_{e}, \alpha_{h}$ for deep traps are about $0.1-10 \mathrm{~cm}^{-1}$. However, for high intensity of signal wave propagating in the waveguide the absorption rates $b_{e s} I_{s}$ and $b_{h s} I_{s}$ can be comparable with $b_{w} I_{w}$ and has to be taken into account. In the case of periodic light intensity distribution the set of equations (2) can be solved by linearization of variables [7-11].

For the light distribution given by (1) it can be assumed that every variable is periodic with the fundamental period $\Lambda=2 \pi / K$ [7-11]. Thus in the steady state regime all variables can be presented in the form of a Fourier series [12]: $V(z)=V_{0}+V_{1} \cos (K z+\varphi)$, where $V_{0}$ denotes the zero-order solution obtained for homogeneous illumination. Using the procedures described in [11] we obtained an analytical solution for the amplitude of the first harmonic of a space charge field expressed in terms of characteristic field: $E_{d h}=K\left(k_{B} T_{L} / q\right)$ and $E_{d e}=K\left(k_{B} T_{e} / q\right)$ which denote diffusion fields, $E_{\mu h}=1 / \tau_{h} \mu_{h} K, E_{\mu e}=$ $1 / \tau_{e} \mu_{e} K, \quad\left(\tau_{e}, \quad \tau_{h}\right.$ designate recombination times for electrons and holes, respectively) and $E_{q}=$ $\left(q / \varepsilon_{0} \varepsilon K\right) \mathrm{r}(1-r) N_{D}$

$$
E_{1}=-m \frac{\left(E_{\mu e}+E_{\mu h}\right) E_{a}+i\left(E_{\mu h} E_{d e}-E_{\mu e} E_{d}\right)}{\left(E_{\mu e}+E_{d e}-i E_{a}\right)\left(1+A_{h} \frac{I_{s}}{I_{o}}\right)+\left(E_{\mu h}+E_{d}+i E_{a}\right) \frac{\mu_{e n}}{\mu_{e}}\left(1+A_{e} \frac{I_{s}}{I_{o}}\right)+\frac{E_{d} E_{d e}+E_{a}^{2}}{E_{q}}}
$$

field and $E_{s c}$ is a space charge field), $I_{w}$ - the light intensity of writing beams, $I_{\mathrm{s}}$ - the light intensity of a signal beam, $\beta$ - the thermal excitation rate, $\gamma$ - the trapping coefficient describing recombination of carriers at the donors traps, $\mu$ - carrier mobility along the quantum wells, $\varepsilon_{0}$ - the permittivity of the vacuum, $\varepsilon$ - the effective dielectric constant of MQW structure, $q$ - the absolute value of the elementary charge, $k_{B}-$ the Boltzmann constant, $T_{L}$ and $T_{e}$ - the absolute temperatures of the lattice and of electrons. Term $b I_{w}$ represents direct
The grating erasing is described in Eq. (3) by $A_{e}=\left(\lambda_{s} / \lambda_{w}\right)\left(\alpha_{e} / \alpha\right)$ and $A_{h}=\left(\lambda_{s} / \lambda_{w}\right)\left(\alpha_{h} / \alpha\right)$, where $\alpha_{e}=s_{e}\left(N_{D}-N_{A}\right), \alpha_{h}=s_{h} N_{A}$ and $\alpha$ are absorption coefficients. The nonlinear electron mobility $\mu_{e n}$ is given by

$$
\mu_{e n}=\frac{\mathrm{d} v_{e}(E)}{\mathrm{d} E}+i E_{d e}\left[\frac{\mathrm{d} \mu_{e}(E)}{\mathrm{d} E}+\mu_{e}(E) \frac{\mathrm{d} \ln T_{e}}{\mathrm{~d} E}\right]
$$


For $I_{s}=0$ the expression describing $E_{1}$ becomes coincident to the corresponding equation in Ref [11]. The dependence of the space-charge field on the signal beam intensity for typical parameters of PMQW [7,9] and various defect densities is presented in Fig.1. The parameters used in calculations are listed in Table 1 . The grating period $\Lambda=100 \mu \mathrm{m}$ is greater than so-called cut-off value $\Lambda_{c}$ [7], which allows to reach the maximum amplitude of a space charge field.

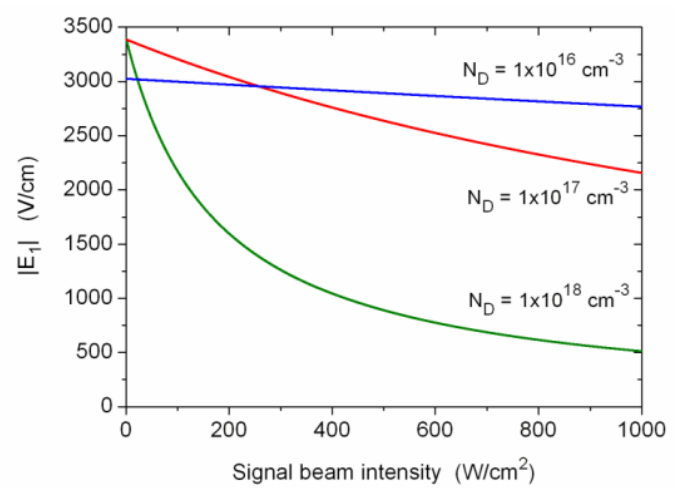

Fig. 1. Photorefractive field amplitude as a function of signal beam intensity for $\mathrm{I}_{0}=1 \mathrm{~W} / \mathrm{cm}^{2}$ and different traps concentrations. The grating period $\Lambda=100 \mu \mathrm{m}$ and material parameters are the same as in Ref. [7].

The signal beam propagating in the waveguide removes carriers from deep traps and in this way erases the photorefractive grating. When the trap concentration decrease the absorption coefficients $\alpha_{e}$ and $\alpha_{h}$ become smaller and the erasing of the grating process is reduced. Therefore to minimize the effect of erasing the concentration of donor defects should be relatively small.

The space-charge field amplitude as a function of a signal wave intensity for defect density of $N_{D}=1 \times 10^{16} \mathrm{~cm}^{-3}$ and various external beams intensities are plotted in Fig. 2.

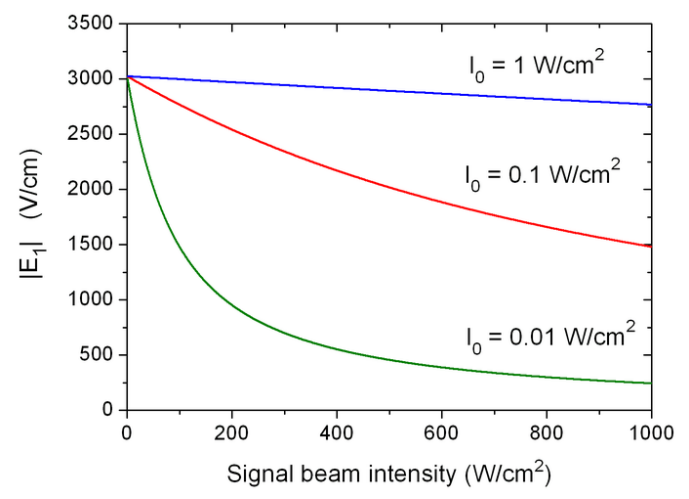

Fig. 2. Photorefractive field amplitude as a function of signal beam intensity for defect density $1 \times 10^{16} \mathrm{~cm}^{-3}$ and different intensities of an external laser beams. The grating period $\Lambda=100 \mu \mathrm{m}$ and material parameters the same as in Ref. [7].
Table 1. Values of parameters used in calculations

$\begin{array}{ll}\text { Writing Wavelength } & \lambda_{w}=633 \mathrm{~nm} \\ \text { Signal Wavelength } & \lambda_{s}=850 \mathrm{~nm} \\ \text { Donors density } & N_{D}=1 \times 10^{16} \mathrm{~cm}^{-3} \\ \begin{array}{l}\text { Compensation ratio of donors traps } \\ \text { (dominance of holes in PR transport) }\end{array} & r=N_{A} / N_{D}=0.8 \\ \text { Fringes contrast } & m \cong 1 \\ \text { External Electric Field } & E_{a}=2.5 \mathrm{kV} / \mathrm{cm} \\ \text { Period of grating } & \Lambda=10 \mu \mathrm{m} \\ \text { Average refractive index of MQW } & n_{\mathrm{f}}=3.55 \\ \text { Resonant absorption coefficient } & \alpha=1 \times 10^{4} \mathrm{~cm}^{-1} \\ \text { Absorption coefficients due to the } & \alpha_{e}=0.02 \mathrm{~cm}^{-1} \\ \text { ionization of defects } & \alpha_{h}=0.8 \mathrm{~cm}^{-1}\end{array}$

The results of calculations show that the use of photorefractive grating for switching signals guided in a semi-insulating MQW is limited to signals of moderate intensities. If the wave confined in the waveguide attains large intensity, the process of grating erasing due to photoionization of deep defects has to be taken into account. In such a case, permanent illumination by external waves is necessary to keep the grating. The erasing rate of the grating can be reduced by using a device with a small density of deep impurities.

The project was funded by the National Center for Science awarded by decision number DEC2011/01/B/ST7/06234.

\section{Reference}

[1] D.D. Nolte, J. Appl.Phys. 85, 6259 (1999).

[2] M. Wichtowski, A. Ziółkowski, E. Weinert-Rączka, J. Opt. 12, 065201 (2010).

[3] E. Weinert-Rączka, M. Wichtowski, A. Ziółkowski, G. Staroń, Acta Phys. Pol. A 103, 229 (2003).

[4] A. Ziółkowski, E. Weinert-Rączka, J. Opt. A: Pure Appl. Opt. 9, 688 (2007).

[5] A. Ziółkowski, J. Opt. 14, 035202 (2012).

[6] M. Wichtowski, E. Weinert-Rączka, Acta Phys. Pol. A 103, 239 (2003).

[7] Q. Wang, R.M. Brubaker, D.D. Nolte, M.R. Melloch, J. Opt. Soc. Am. B 9, 1626 (1992).

[8] Q. Wang, D.H. Olson. G.E Doran, W.H. Knox, A.M. Glass, J. Opt. Soc. Am. B 7, 2217 (1990).

[9] D.D. Nolte, M.R. Melloch, Photorectractive effects and Materials, ed. by D.D. Nolte (Kluwer, Dordrecht 1995).

[10] L.F. Magaňa, F. Agulló-López, M. Carrascosa, J. Opt. Soc. Am. B 11, 1651 (1994).

[11] Q. Wang, R.M. Brubaker, D.D. Nolte, J. Opt. Soc. Am. B 9, 1773 (1994).

[12] P. Vaveliuk, B. Ruiz, N. Bolognini, Phys. Rev. B, 59, 10985 (1999).

[13] D.S. Chemla, D.A.B. Miller, P.W. Smith, A.C. Gossard, W. Wiegmann, IEEE J. Quantum. Electron. 20, 265 (1984).

[14] D.D. Nolte, Q. Wang, Appl. Phys Lett. 58, 2067 (1991). 